\title{
DER FORTBILDUNGSKURS \\ DES VEREINS SCHWEIZERISCHER GYMNASIALLEHRER
}

\author{
vom 13. bis 19. Oktober 1946 in Lausanne
}

Von Paul Vosseler

An dem vom Schweizerischen Gymnasiallehrerverein veranstalteten Fortbildungskurs organisierte der Verein Schweizerischer Geographielehrer eine Serie instruktiver Vorträge und Exkursionen.

Über Neuerungen auf dem Gebiete des Kartenwesens sprach Ed. Iмног. Neue Formen des Vermess ung w es ens brachte die Photogrammetrie. Durch Doppelaufnahmen, sei es von der Erde oder vom Flugzeug aus, kann die Erdoberfläche stereometrisch erfaßt werden, und mittels komplizierter Auswertegeräte lassen sich Karten und maßstabsgetreue Profile und Ansichten konstruieren. Schon heute werden unbekannte Länder auch mit Anlehnung an wenige bestimmte Punkte topographisch und geologisch aufgenommen, und für die Zukunft wird in der Radartriangulation wohl eine Methode entwickelt, die erlaubt, große unbekannte Gebiete lufttrigonometrisch zu vermessen. Zur Erzeugung guter Bilder werden neuerdings Infrarot- und Farbaufnahmen verwendet. Die Methode der Photogrammetrie verringert wesentlich die Zeit und die Kosten der Aufnahmetätigkeit und ermöglicht die Auswertung der Bilder im Arbeitsraum. Besonders Flugaufnahmen bilden ein prächtiges Anschauungsmaterial für das Studium physio- und anthropogeographischer Verhältnisse, namentlich durch Stereoskopbetrachtung und Reproduktion in Anaglyphen, die bei der Betrachtung durch eine Rot-Grün-Brille ein plastisches Bild ergeben. Die Landkartenherstellung war von jeher abhängig von der Drucktechnik. Der Holzschnitt des sechzehnten Jahrhunderts brachte grobe, doch schöne Kartenbilder; beim Kupferstich entwickelte sich die Schraffenkarte. Der Lithographie verdanken wir bei Verwendung verschiedener Farbplatten die Reliefkarte. Neuerdings bringt die photomechanische Übertragung farbiger Kartenbilder eine Umwälzung in der Reproduktionstechnik, besonders durch die Anwendung der Farbenphotographie.

In vierzig Prozent des Mittellandes ist die Grundbuchvermessung durchgeführt. Die Grundbuchübersichtspläne sind eine unumgängliche Grundlage der neuen Landeskarte. Diese wird im Alpengebiet ergänzt durch die militärische Landesvermessung. Die private Kartographie erstellte zwölf neue Kantonskarten und arbeitet an der Jubiläumsausgabe des schweizerischen Mittelschulatlasses. Ein besonderer Zweig der Kartographie ist der Faksimiledruck wertvoller alter Schweizer Karten wie die ihrer Zeit vveit vorauseilenden Zürcher Karte von Konrad Gyger.

Als Einleitung zu einer Stadtwanderung gab $\mathrm{CH}_{\mathrm{H}}$. BIERMANN einen Überblick über die Lage, die Entwicklung und das Wesen von Lausanne. Die ältesten Spuren der Stadt reichen in die Römerzeit, wo in der Nähe des Flon-Deltas bei Vidy die Reste einer Handelssiedlung Lousanna ausgegraben wurden. Im siebenten Jahrhundert hat sich das Zentrum der Stadt, der Bischofssitz, auf einen Molassesporn $z$ wischen den Bachschluchten der Loue und des Flon verschoben, wo im dreizehnten Jahrhundert die Kathedrale, ein prächtiger frühgotischer Bau, entstand, dem Kirchen des achten und elften Jahrhunderts vorangingen. Mit einem wundertätigen Marienbild war sie bis zur Reformationszeit Wallfahrtskirche. Zwei Klöster, Sainte-Maire und Saint-Etienne, schlossen nach $\mathrm{N}$ und $\mathrm{S}$ die Cité ab, in der die Berner im sechzehnten Jahrhundert die Akademie gründeten, die als wuchtiger Renaissancebau den W-Rand des Riegels krönt. Neben der Hochstadt war auf dem den Flon in O- W'-Richtung ab- 
drängenden Seitenmoränenwall des Rhonegletschers und in der Flon-Niederung eine Unterstadt entstanden, die mit vier Quartieren und Vorstädten längs Ausfallstraßen noch lange als Gemeinde seJbständig blieb. Saint-François und Madelaine waren die kirchlichen Zentren. Die Stadt, welche vom dreizehnten bis achtzehnten Jahrhundert rund achttausend Einwohner zählte, war eingebettet in die Rebberge des Genferseehangs und besaß keine wesentliche Industrie. 1803 wurde sie Hauptort eines Kantons, und seither entwickelte sich hier neues Leben. Um die Verkehrsverhältnisse zu bessern, wurde 1830 der Grand Pont über das Flontal gebaut, und zu Beginn unseres Jahrhunderts folgten weitere Hochbrücken. Außerhalb der Stadt wurde 1856 auf steilem Hang der Bahnhof angelegt, so daß die Hauptverkehrsanlagen gegen Renens hinausrücken mußten. Die Verkehrsverbesserung brachte den Aufschwung als Bildungsstätte und Fremdenort; Kennzeichen sind zahlreiche Unterrichtsgebäude der Universität sowie staatlicher und privater Schulen.

Ein Gang durch die Altstadt mit der hochthronenden Cité, der eng im Tal sich scharenden Unterstadt, den auf dem Moränenrücken sich drängenden Gäßchen und dem belebten Zentrum der Geschäftscity um Saint-François, zu den Lagerhäusern, die den aufgefüllten Talboden des Flontales überziehen, und zu der aussichtsreichen Terrasse von Montenon, wo der Blick die von Pärken durchsetzten Hauszeilen bis zum Hafenquartier von Ouchy streifte, zeigte die vielgestaltige Stadt mit dem Gemenge ältester Stadthäuser und protziger Bauten aus der Jahrhundertwende, mit den markigen Akzenten der spitzen Kirchturmhelme.

In seinem Vortrag "Géopolitique et géographie politique» setzte sich $\mathrm{C}_{\mathrm{H}}$. BurkY mit zwei anscheinend ähnlichen Richtungen der Anthropogeographie auseinander.

Die der Großraumpolitik Deutschlands dienende Geopolitik ging bei der Erklärung der Staatsbildung in erster Linie von den Raum- und Lagebeziehungen aus, dann auch von dem Menschen, seiner Verteilung und Zivilisation. Doch der Mensch beeinflußt die Natur, er schafft eine Wirtschaft, die Grundlage der Existenzbedingungen und des Lebensstandards, und die Beziehungen der verschiedenen Wirtschaften bedingen die Politik der Völker. Die politische Geographie ist ein wichtiger Zweig der Allgemeinen Geographie, als Vermittlungswissenschaft zwischen Natur- und Geisteswissenschaften, und damit viel umfassender als die Geopolitik.

Hierauf entwarf R. Meylan ein Bild der Vallée de Joux, jener reizvollen Juralandschaft, welche er in seiner Dissertation beschrieben hat. Die anschließende Exkursion vermittelte einen guten Einblick in diese Hochjuralandschaft. Bei Sonnenschein, der den Laubwald mit rotem Licht übergoß, fuhr man durch die großen Waadtländer Dörfer am Jurarand, erklomm in zahlreichen Kehren die Höhen des Marchairuzpasses, von wo aus den Sêches d'Amburnex ein Besuch abgestattet wurde. Dort umrahmt waldumgebenes Weideland einer Polje ein Karstgebiet mit niedern verkrüppelten Fichten eines Frostloches, die die zahlreichen Karren, Dolinen und Höhlen kaum unter ihren Wurzeln verstecken. Der Abstieg ins Jouxtal eröffnete einen prächtigen Einblick in die Kulturoase inmitten der großen Waldgebiete, die sich im $\mathrm{N}$ des Tales zum Grenzsaum des mächtigen Risouxwaldes zusammenschließen. Die schimmernden Seen gehen nach oben in Moorland und breite Mattenflächen über, durch welche sich die Orbe schlängelt. Große Dörfer mit schindel- und blechgedeckten Jurahäusern, mit stattlichen Fabriken und Arbeiterhäusern scharen sich längs den Talseiten. Ein Besuch der Uhrmacherschule in Le Sentier zeigte die Bedeutung dieser arbeitsintensiven Industrie für das Tal. Eine Wanderung an den Lac des Brenets, zu der Stromquelle der Orbe und eine abendliche Fahrt über Romainmôtier, wo die Kunst früherer Jahrhunderte in der prächtigen Abbatiale auf uns wirkte, schlossen die Exkursion.

Über Meteorologie in der Schule berichtete E. LeEmanN. Die Wetterbeobachtung eignet sich wie wenige Zweige des Geographieunterrichts als Anleitung 
der Schüler zu selbständiger Arbeit. Hier können die Grundlagen für das Verständnis des Klimas gewonnen werden. An den jeweiligen Wetterlagen lassen sich die Elemente der Meteorologie erfassen: Druck- und Wärmeverhältnisse als Energiefaktoren, Feuchtigkeit, Niederschläge und Wolkengestalt. Diese Beobachtungen bieten für die Lektüre der Wetterkarte, deren Verständnis namentlich im Hinblick auf die Wettervorhersage unbedingt gefordert werden sollte, die Grundlage.

In seinem Referat über Anschauungsmittel im Geographieunterricht betonte P. Vosseler die Wichtigkeit der Beobachtung als Grundlage für das geographische Denken. Da den Exkursionen im Lehrplan nur wenig Raum eingeräumt ist und so nur eine beschränkte Anzahl von Begriffen in der Natur selber erworben werden kann, müssen Anschauungsmittel ergänzend verwendet werden. Solche bestehen in der Wandtafelskizze, in vereinfachten Karten und Profilen, vor allem in Blockdiagrammen. Wichtig ist auch das Bild, als Wandbild, wie es neuerdings das Schweizer Schulwandbilderwerk schafft, als Lichtbild, dessen Auswahl der Verein Schweizerischer Geographielehrer förderte. Die Farbenphotographie rollt die Frage des Formats auf, indem neuerdings das Kleinformat gefordert wurde. Ein hervorragendes Anschauungsmittel ist auch das Relief als dreidimensionale Darstellung der Landschaft. Besonders für den Geologieunterricht ist das Relief zur Einführung und Vertiefung unumgänglich, da die spärlichen Aufschlüsse in der Natur nicht genügen, dem Schüler den Bau eines Gebietes verständlich zu machen. Der Vortragende wies auf die von ihm hergestellten Typenreliefs hin, die großmaßstäbig genug, doch handlich sind, um jederzeit im Unterricht verwendet werden zu können. Ahnliche Bedeutung wie das Relief, wenigstens für die Demonstration von Bodenformen, besitzt der Sandkasten. Als neues Lehrmittel hat sich der Lehrfilm eingeführt, der durch die Schweiz. Arbeitsgemeinschaft für Unterrichtskinematographie (SAFU) und durch die kantonale Lehrfilmstelle von Baselstadt gefördert wird. Er ermöglicht die Darstellung der Bewegung, die durch Trickfilme, Zeitlupe und -raffer vereinfacht und analysiert werden kann.

E. Nussbaum gab in seinem Vortrag "Forderungen der Hochschule an den Geographieunterricht an Mittelschulen» vorerst einen historischen Überblick der Ansichten hervorragender Pädagogen über die Eignung des Geographieunterrichts für die Jugendbildung. H. Pestalozzi und Fellenberg, K. Ritter und A. von НuмвоLDт betrachteten die Erdkunde, vor allem die Heimatkunde, als zentrales Bildungsfach. Erst um die Jahrhundertwende erfolgte jedoch durch die Richthofenschule, die das Antlitz der Erde aus physiogeographischen Grundlagen zu erfassen suchte, ein eigentlicher Aufschwung. Doch befindet sich unser Fach noch immer in einer Kampfstellung, und die Einsicht, daß nur der Besitz eines umfassenden Weltbildes, das sich auf das Wissen physio- und anthropogeographischer Tatsachen und ihrer Verknüpfung stützt, das auch die Grundlage einer staatsbürgerlichen Erziehung darstellt, ist noch nicht bei allen Schulmännern durchgedrungen. Nussbaum präzisierte dann die Forderungen der Hochschulen an den Geographieunterricht der Maturitätsanstalten wie folgt: Sichere Kenntnisse der geographischen Tatsachen der Schweiz und damit ein Vertrautsein mit deren Topographie; klare Vorstellungen der geographischen Lage der Erdteile und der Nachbarländer der Schweiz; Einblick in die Zusammenhänge zwischen Natur und Kultur, entsprechend der Aufnahmefähigkeit des Schülers.

Zum Abschluß des instruktiven Kurses sprach H. Gutersorn über den Bildungswert der Geographie. Die Aufnahmefähigkeit des Schülers auf der Volksschulstufe beschränkt den Stoff auf die Aneignung topographischen Wissens und auf die Erarbeitung einfacher Tatsachen. Doch können durch die Betrachtung der Landschaftselemente schon hier einfache Zusammenhänge zwischen Natur- und Menschenwerk erkannt werden. Die Erweiterung dieser Gedankenarbeit ist der Mittelschule vorbehalten, jedoch ermöglicht erst die Hochschule das Verständnis in alle Grundlagen und ihr Zusammenwirken bei der Gestaltung einer Landschaft. Die Geographic dringt ein in 
die Biologie der Landschaften, die in der Art und in der Mannigfaltigkeit der wirkenden Faktoren sehr verschieden sein kann. Birgt schon die Erfassung der geographischen Erscheinungen eine große Gedankenarbeit, wie viel intensiver ist erst die geistige Betätigung bei dem Versuch, die Erscheinungen zu generalisieren. Dazu braucht es geistige Reife, die sich erst auf der Oberstufe der Mittelschule langsam entwickelt. Deshalb muß auch der Geographieunterricht, soll er bereits auf der Mittelschulstufe eine geistige Durchdringung des materiellen Wissensstoffes ermöglichen, bis in die obersten Klassen durchgeführt werden. Denn erst diese geistige Durchdringung kann zur Erfassung der Harmonie einer Landschaft führen, aber auch zur Erkenntnis der Störung dieser Harmonie, der die Landesplanung Einhalt zu gebieten sucht.

Ein die Tagung abrundender Ausflug auf einem der schönen Genferseedampfer führte alle Teilnehmer zum Schloß Chillon. Das Schloß ist auf einer kleinen Insel erbaut, der Fortsetzung eines Doggerkalkkammes des Nordschenkels der Rochersde-Naye-Synclinale. Diese stößt hier mit Felsabstürzen und steilen, von Laubwald bedeckten Schutthängen auf einer Länge von rund einem Kilometer ans Seeufer vor und trennt scharf die von Moränen überdeckten terrassierten Hänge von den breiten Schuttkegeln der Umgebung von Montreux sowie den Ausräumungen und den Aufschüttungen des Tinière-Wildbaches am Rand der untersten Rhoneebene. Von tiefgründigem Verwitterungsboden und klimatischer Gunst der Spalierlage und dem mildernden Einfluß des Seebeckens zeugen die bis sechshundert Meter hoch steigenden Reben südlich und nördlich des Gebirgsvorsprungs. Von der ausgezeichneten Verkehrslage am Zugang zu einem der am frühesten begangenen Alpenpässe erzählen die städtischen Siedlungen am Seeufer: Vevey, Tour-de-Peilz und Villeneuve, aber auch die modernen Hotelzeilen, und nicht von ungefähr ersteigen steile Bergbahnen die umgebenden Terrassen und Aussichtsberge, von deren Höhen Gruppen großer Hotels grüßen. In diese Landschaft des Fremdenverkehrs ist heute Chillon einbezogen. Allerdings haben andere Gründe zum Bau des Schlosses geführt. Die Sperrlage am Zugang zum Wallis, die ja in der Gegenwart abermals einer Befestigung der Talflanken rief, die Schutzlage auf einem Inselsporn, die zentrale Lage inmitten einer dicht besiedelten Landschaft veranlaßte hier die Herren des Genfersees und des Wallis zur Burggründung. Mit dem Fallen der territorialen Macht versank dann allerdings die Bedeutung der einstigen zentralen Verwaltungsstätte. Als Museum aber wird sie mit ihren riesigen Hallen, bunten Zimmern und tiefen Verließen immer wieder Besucher anziehen; bildet doch der prächtige Bau in seiner Lage über den blauen Wellen des Sees, vor der Silhouette der hohen Kalkberge, den Mittelpunkt einer der schönsten Gegenden der Schweiz. Es war eine gute Idee, den aus der ganzen Schweiz hergeströmten Teilnehmern des Kurses dieses Kleinod zu zeigen - als Krönung einer Fahrt über den Genfersee und ihnen dort zugleich die waadtländische Gastfreundschaft zu beweisen. Denn ein idealerer Ort, wo Fachgenossen inmitten einer grandiosen Natur- und Kulturlandschaft Erfahrungen austauschen, wo alte Freundschaften erneuert, neue geschlossen werden konnten, hätte nicht gewählt werden können.

\section{RÉSUMÉ}

L'auteur donne un compte rendu sur le cours de perfectionnement de la Société suisse des professeurs de géographie donné du 13 au 19 octobre 1946 à Lausanne. E. Imнiof parla des progrès en cartographie. Сh. Biermann donna un aperçu sur un guide de Lausanne. Сн. Burky analysa la géopolitique et la géographie politique. R. Meyzan expliqua la Vallée de Joux, qui fut visitée par une excursion. P. Vosseler montra des possibilités nouvelles de représentation en géographie. F. Nussbaum développa les obligations que les universités ont en ce qui concerne l'enseignement de la géographie dans les écoles secondaires, et H. Gutersohn traita la valeur éducatrice de la géographie. Une excursion au château de Chillon termina ce congrès instructif. 


\section{R I A S S U NTO}

L'autore dà un resoconto delle conferenze e delle escursioni tenutc al corsu di perfezionamento della Società svizzera dei professori di geografia avuto luogo dal 13 al 19 ottobre 1946 a Losanna. E. IMHof parlò sui progressi conseguiti nel campo della cartografia. CH. BisRmanN dimostrò la città di Losanna. CH. Bur KY analizzò le relazioni esistenti tra geopolitica e geografia politica. R. MeYLAN illustrò la Vallée de Joux che fu visitata in un'escursione. P. Vosseler parlò sulle possibilità delle rappresentazioni grafiche nell'insegnamento. F. NussBaum espose gli obblighi delle università nei riguardi dell'insegnamento della geografia nella scuola media e da ultimo H. GuTERsohn analizzò il valorc educativo della geografia. Una cscursione al castello di Chillon chiuse questo congrcsso istruttivo.

\section{NEKROLOGIE}

\section{Paul Joachim Haffter-Bryner 1869 - 1946}

Am 17. April 1946 entschlief in Zürich nach langem Leiden PAUL J. HAFFTER-BryNER im 77. Lebensjahr. Mit ihm ging ein stiller Privatgelehrter und treuer Freund der Naturwissenschaften in die ewige Ruhe ein. Paul Haffter, ein Bürger von Weinfelden, wurde am 2. November 1869 in Meilen geboren. In Zürich verlebte er eine schöne, ungesorgte Jugend; am zürcherischen Gymnasium bestand er das Maturitätsexamen. Innere Neigung und eine eher schwächliche Gesundheit bewogen ihn zum Studium der Landwirtschaft. Er absolvierte das damalige Eidgenössische Polytechnikum in den Jahren 1888-1892; ein Studiensemester in Bonn schloß sich an. 1893 siedelte der junge Landwirt mit seinen Eltern nach Schloß Berg über, unweit Weinfelden; ex übernahm dort die Leitung des Gutsbetriebes. Neben der praktischen Betätigung fand er stets Zeit für die Botanik, für die er durch seinen Lehrer C. SCHRÖtER gewonnen wurde. Botanik und Ethnographie waren die Lieblingsgebiete, denen er seine freien Stunden schenkte und für die er eine ursprüngliche wissenschaftliche Begabung besaß.

Nach zwanzigjähriger praktischer Betätigung in Berg verkaufte unser Verblichener sein Gut, um nunmehr seinen geliebten Wissenschaften zu leben. Das Oberengadin wurde zu seiner zweiten Heimat. Er durchstreifte zusammen mit seiner gleichgesinnten Gattin dieses Juwel schweizerischer Landschaft und erwarb sich dort eine ausgezeichnete Kenntnis der alpinen und subalpinen Flora. Ein dauernder freundschaftlicher Kontakt mit C. SCHRÖTER und später mit dem Pflanzensoziologen J. Braun-BLANQUET vermittelte ihm wissenschaftliche Anregungen und vertiefte Kenntnis. In Zusammenarbeit mit dem Bodenkundler bearbeitete P. J. HAFFTER pflanzensoziologisch und bodenkundlich besonders die Wald- und Zwergstrauchgesellschaften des Oberengadins. Eine erste größere Arbeit darüber erschien 1933 in den "Berichten der Schweizerischen Botanischen Gesellschaft». Ein großes Material über die Soziologie und Oekologie anderer Zwergstrauchgesellschaften der subalpinen Stufe liegt noch in Manuskriptform vor; ein Teil wurde im "Prodromus der Pflanzengesellschaften" (Faszikel 6), Klasse dcr Vaccinio-Piceetea, von J. Braun-Blanquet, G. Sissingh und J. Vlieger 1939 verwertet.

Zusammen mit seiner Gattin unternahm P. J. HAFFTER alljährlich weite Reisen, die um den ganzen Erdball führten. Vom hohen Norden bis zum Kap Horn, nach Indien, China, Japan führten sie. Holländisch-Indien, Australien und die Südseeinseln waren mehrmals das Ziel seiner Studienfahrten. Afrika wurde kreuz und quer bereist. Auf diesen weiten Fahrten erwarb er sich eine erstaunliche Kenntnis der Flora und der Völker fremder Länder. Seine Freunde bedauern, daß der stille, in sich gekehrte Privatgelehrte vor lauter Erkennen nicht zum schriftlichen Bekennen kam, so daßdie wissenschaftliche Gemeinde die Früchte seines Schaffens missen muß. Seinen wissenschaftlichen Freunden, dazu zählten auch F. SAR ASIN und der Basler Botaniker SENN, erschloß er sich, ihnen wurde er zum reichen Quell von Anregungen.

Mit Paul HaffTer ist ein großer Naturfreund und Naturkenncr von uns geschieden. Die ihn kannten, bewahren ihm ein freundschaftliches, treues Andenken.

H. PALLMANN

\section{VERBANDSTÄTIGKEIT - ACTIVITÉ DES SOCIÉTÉS}

Aktion Bauernhausforschung in der Schweiz. Ziel der Aktion, die durch die Schweizerische Gesellschaft für Volkskunde vor zwei Jahren ins Leben gerufen wurde, ist eine möglichst umfassende, -gleichartige und technisch einwandfreie Bestandesaufnahme der Bauernhausformen unseres Landes. Das gesammelte Material soll schließlich in einem Quellenwerk publiziert und der Forschung zur Verfügung gestellt werden. Es dient aber nicht nur dieser, sondern auch den Kreisen und Institutionen, die praktisch am Heimatschutz, am Landschaftsschutz, an der Denkmalpflege und an der Gestaltung bäuerlicher Wirtschaft und bäuerlicher Kultur interessiert sind.

Kürzlich sind im Kanton Zürich ein Kantonalkomitee und ein Arbeitsausschuß für Bauernhausforschung gegründet worden, nachdem andere Kantone bereits an die Arbeit gegangen sind und nachdem der Technische Arbeitsdienst in der letzten $Z$ wischenkriegszeit wertvolle Vorarbeit geleistet hat. Die Aufnahmen in den Kantonen, denen eine Inventaraufnahme zurFeststellung dergeeignetenObjekte vorangeht, werden nach einheitlichen Leitlinien durchgeführt. Leiterder gesamtschweizerischen Aktion ist A. BAESCHLIN, Architekt BSA., Schaff hausen, der in Spanien auf dem Gebiete der Hausforschung gearbeitet hat und in zwei Jahren in der Schweiz einen internationalen Kongreß für Hausforschung durchzuführen gedenkt. R. WrisS 